Journal of Research in Interprofessional

Practice and

Education

Vol. 2.3

Editorial

August, 2012

\section{Journal of Research}

in Interprofessional Practice and Education (JRIPE)

Vol. 2.3 Editorial

(c) 2012

Corresponding author:

Hassan Soubhi

hassan.soubhi

@usherbrooke.ca

\section{Editorial: Complex Care and the Need for Collaborative Brains}

\author{
Hassan Soubhi, MD, PhD
}

With the rising complexity of patient care comes a rising need for collaborative work. The articles in this issue explore two important questions in this regard: How should we organize collaborative work? And how should we prepare future generations for it?

Packard et al. [1] test whether students' performance working up a case and perceptions of interprofessional skills would improve if they are given modeled examples of interprofessional communication and a team reasoning framework.

Holmqvist et al. [2] highlight the need for co-ordinated research efforts to determine the usefulness of student-run clinics as ideal sites to advance learning in teamwork and social accountability.

Newhouse et al. [3] add to our understanding of how to design effective heart failure management programs. Using a Delphi process, they report data from a series of consultations among health professionals, patients, and family caregivers.

Hutchison et al. [4] test the hypothesis that clinical pharmacists, physicians, and other healthcare professionals providing medication therapy management can improve outcomes and reduce costs among patients at high risk of adverse reactions from medication.

Arar et al. [5] provide insights into the cycles of growth through which multidisciplinary research teams operate. And finally, Butson et al. [6] examine the problems that can affect the creation of interprofessional virtual communities of practice.

All of these studies reflect variations on the theme of care complexity and the need for multidimensional platforms to address that complexity. These studies also highlight the kinds of skills we need to hone-dialogue, compassion, attention to the other, extending loyalties, and social interdependencies. Indeed, when care is complex, when interactions among health professionals and patient become intricate, with the need to process higher amounts of information, it pays to hone our collaborative brains to broaden our perspective, widen our insights, and extend our predictive abilities. Such collaborative brains would work their way, through successive approximations, toward a better understanding of interprofessional practice. Armed with ideas, tools, and vocabulary, they would elevate their approaches to problems-each new understanding building on another, each new insight the source of a productive vitality that can carry the group for a while, until the next problem, the next challenge to its imagination, the next insight. 


\section{JRIPE \\ Journal of Research in Interprofessional Practice and Education}

249

Editorial

Hassan Soubhi

\section{References}

1. Packard, K., Chehal, H., Maio, A., Doll, J., Furze, J. Huggett, K, Jensen, G., Jorgensen, D., Wilken, M., \& Qi, Y. (2012). Interprofessional Team Reasoning Framework as a Tool for Case Study Analysis with Health Professions Students: A Randomized Study. Journal of Research in Interprofessional Practice and Education 2(3), 250-263.

2. Holmqvist, J., Courtney, C., Meili, R., \& Dick, A. (2012). Student-Run Clinics: Opportunities for Interprofessional Education and Increasing Social Accountability. Journal of Research in Interprofessional Practice and Education 2(3), 264-277.

3. Newhouse, I., Heckman, G., Harrison, D., D’Elia, T., Kaasalainen, S., Strachan, P.H., \& Demers, C. (2012). Barriers to the Management of Heart Failure in Ontario Long-Term Care Homes: An Interprofessional Care Perspective. Journal of Research in Interprofessional Practice and Education 2(3), 278-295.

4. Hutchison Jr., R.W., Hash, R.B., \& Nault, E.C. (2012). Multidisciplinary Team of a Physician and Clinical Pharmacists Managing Hypertension. Journal of Research in Interprofessional Practice and Education 2(3), 296-302.

5. Arar, N.H., \& Nandamudi, D. (2012). Advancing Translational Research by Enabling Collaborative Teamwork: The TRACT Approach. Journal of Research in Interprofessional Practice and Education 2(3), 303-319.

6. Butson, R., Hendrick, P., Kidd, M., Brannstrom, M., \& Medberg, M. (2012). Developing a Virtual Interdisciplinary Research Community in Clinical Education: Enticing People to the "TeaRoom." Journal of Research in Interprofessional Practice and Education 2(3), 320-338. 